\title{
Follow-up for patients with malignancies after radical treatment: unresolved questions
}

\author{
Vladimir Moiseyenko
}

Received: 27 May 2014 / Accepted: 27 May 2014 / Published online: 3 July 2014

(C) Springer-Verlag Wien 2014

Contemporary clinical practice is supported by a variety of international evidence-based standards (NCCN, ASCO, ESMO, NCI, NICE, etc.) that determine optimal approach for patients with malignancies after the carried out treatment. Essentially, they can be divided into following groups:

1. Identification of disease recurrence in patients with radical treatment for the subsequent effective therapy (anal cancer, colorectal cancer, sarcomas, lymphoproliferative disorders, germ cell tumors),

2. monitoring of the necessity of interventions in patients with palliative treatment to maintain appropriate quality of life,

3. monitoring of possible distant treatment complications (function of a thyroid gland after radiation therapy at head/neck tumors, the nutrition of patients after gastrostomy) and

4. identification of second or multiple primaries (lung cancer: $1-9 \%$, at head/neck tumors: $3-5 \%$ ).

The problem of carrying out the appropriate follow-up (FU) for cancer survivors and those who recently passed through palliative phase is inevitably increasing in the medical system with the growing frequency of cancer burden caused by the steady rise of malignant tumor incidence and by the success of available treatment options. However, the greatest difficulties are caused by the frequency of visits and examination volume after the active treatment has been finished.

Prof. Dr. V. Moiseyenko, MD $(\varangle)$

Diagnostic and Treatment Center under International Institute for Biological Systems named after S.M. Berezin,

43, Karla Marksa str., Pesochny,

197758 St. Petersburg, Russia

e-mail: moiseyenkov@gmail.com, moiseyenkov@icloud.com
Now, it is obvious that treatment, as well as the FU approach, should be individualized urgently. The schedule and structure of the FU procedures should differ by biologic considerations (tumor morphology, disease stage, tumor grade, sensitivity to conventional treatment, median time of relapse after treatment, recurrence frequency, and sites), medical considerations-opportunities of radical treatment in case of disease recurrence (effective cytotoxic drugs or the target therapy, relatively radical second-look surgical procedures, and radical schedules of radiation), and unfortunately also social and economic considerations (geographical region, specialization of center, personnel who carries out FU, and accessibility of infrastructure to carry out the appropriate examinations).

Anyway, FU means a certain interval between visits to specialist and/or nurse of the oncology center, anamnesis, physical examination, laboratory analyses and markers (CEA, etc.), thorax X-ray, ultrasonography of lymph nodes and of abdominal cavity, and some additional methods (computed tomography (CT) scan with/without intravenous contrasting, magnetic resonance imaging (MRI), positron emission tomography (PET), and biopsy in case of suspicion of recurrence).

Obviously, active and frequent supervision is necessary in patients with potentially curable tumors (germ cell tumors and hematologic malignancies) to reveal disease recurrence and conduct effective and probably curative treatment. For example, S. Rotchschild mentioned that available data shows that maximum treatment effect in $15-20 \%$ of patients with anal cancer is registered in week 18 after the treatment was finished; also is mentioned that one-third of the patients show complete response in week 15 after treatment, despite preservation of a residual tumor in week 11. The early biopsy (till 6 months after the end of treatment) is required only in a limited group of patients-in case of large tumors (T4), clinical progression of the disease, or total absence of effect 
from treatment. While PET role in the FU of this group of patients and criteria of metabolic activity of recurrence is discussable, still the question of optimal schedule is open due to the possible toxicities of frequent examinations, for example, from CT or PET. As for other solid tumors, today, there is no evidence-based data indicating that active FU may influence overall survival $(1,2)$. It means that overall survival is identical, notwithstanding the fact that the patient has symptoms or clinical signs of recurrence or not.

In some cases, the appearance of clinical symptoms is the main marker of recurrence. Additional methods of examination, such as MRI, CT, or a metabolic assessment on PET, demand detailed studying, including carrying out randomized prospective clinical trials.

Besides the positive impact, active FU may also have some negative points to consider-additional psychological load for patients during their visits to oncology centers, meaningful risk of the false-positive interpretation of results, invasive diagnostic methods used in particular situations, and financial burden on the health system.

According to the aforementioned facts and summarizing the reviews selected for this issue, FU in both solid and hematologic malignancies after primary treatment represents a topic of great interest. In detailed review of contemporary FU guidelines, authors analyze available clinical data, focusing on the role of modern examination techniques and methods such as PET or CT. Besides the influence of full cytoreduction of recurrence on overall survival does not raise doubts for colorectal cancer or sarcomas with these patients being the appropriate can- didates for close FU, the situation is not that clear for lung cancer and head/neck tumors.

\section{Conflict of interest}

The author declares that there is no conflict of interest.

\section{References}

1. Virgo KS, McKirgan LW, Caputo MC, Mahurin DM, Chao LC, Caputo NA, Naunheim KS, Flye MW, Gillespie KN, Johnson FE. Post-treatment management options for patients with lung cancer. Ann Surg. 1995;222(6):700-10.

2. Younes Z, Johnson DA. Congenital esophageal stenosis: clinical and endoscopic features in adults. Dig Dis. 1999;17(3):172-7.

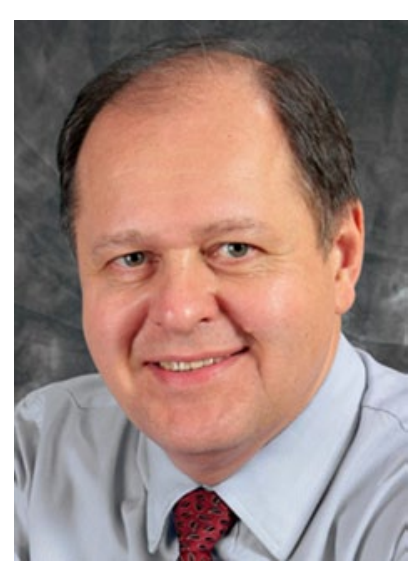

Prof. Dr. Vladimir Moiseyenko, MD 